\title{
Effects of time pressure on context-sensitive property induction
}

\author{
Patrick Shafto \\ Massachusetts Institute of Technology, Cambridge, Massachusetts \\ AND \\ JoHN D. COLEY AND DAVID BALDWIN \\ Northeastern University, Boston, Massachusetts
}

\begin{abstract}
Past research suggests that category-based induction flexibly draws on different kinds of knowledge in different contexts, and that different kinds of knowledge may differ in accessibility. The present study investigates the degree to which knowledge accessibility mediates context-sensitive induction by examining the effects of time pressure on inferences about novel properties of animal species. Participants were told about a novel gene or a novel disease that was true of one category of animals, then rated the likelihood that taxonomically, ecologically, and unrelated animals had the same property, under speeded or delayed conditions. Property effects were observed for taxonomically related species independent of time pressure, but were only observed for ecologically related species in the delayed condition. These results suggest that time pressure selectively restricts access to ecological knowledge, and that knowledge access is critical for context-sensitive inductive reasoning.
\end{abstract}

One remarkable aspect of human intelligence is the ability to reason flexibly based on context. For example, if told that ducks have a novel gene, one might be willing to infer that a taxonomically related animal such as vultures would also have the gene, but an ecologically related animal, such as beavers, would not. However, if told that ducks have a novel disease one might be more willing to infer that beavers and other ecologically related animals might also be susceptible. Underlying these qualitatively different patterns of inference are two different kinds of knowledge about the domain of animals: knowledge about taxonomic relations relevant to the distribution of genes, and knowledge about ecological relations relevant to the distribution of diseases. Context-sensitive property induction depends on the ability to call upon different kinds of knowledge to support reasoning about different kinds of properties.

Psychologists have empirically verified that reasoning in category-based induction tasks flexibly draws upon different kinds of knowledge in different inductive contexts (e.g., Coley, Vitkin, Seaton, \& Yopchick, 2005; Heit \& Rubinstein, 1994; Ross \& Murphy, 1999; Shafto \& Coley, 2003; Shafto, Kemp, Baraff, Coley, \& Tenenbaum, 2005). For example, Heit and Rubinstein (1994) showed that reasoning about novel anatomic properties is predicted by taxonomic similarity alone, but reasoning about novel behavioral properties is predicted by both taxonomic and behavioral similarity. Similarly, in the domain of foods, Ross and Murphy (1999) showed that reasoning about novel nutrients is predicted by taxonomic relations among foods whereas reason- ing about novel social uses is predicted by script relations. These results show that across domains, context-sensitive reasoning is guided by many different kinds of knowledge.

However, not all knowledge in a domain is equally accessible. For example, Ross and Murphy (1999) showed that taxonomic categories (e.g., meat) are more accessible than script categories (e.g., dinner foods) for foods (e.g., steak). In one experiment, priming a food's taxonomic category had no effect on the speed or accuracy of category verification relative to neutral priming, whereas priming a food's script category increased both speed and accuracy of verification. More recently, Vitkin and Coley (2005) have shown that unprimed judgments of taxonomic category membership are faster and more accurate than similar judgments of script category membership. These results suggest that though many kinds of domain knowledge may coexist, they may vary in accessibility, and some kinds of knowledge - in particular, taxonomic knowledge - may have a privileged status (see also Barsalou, 1982).

We have argued that context-sensitive reasoning depends on the ability to call upon different kinds of knowledge, and that different kinds of knowledge vary in their relative accessibility. If so, then manipulating accessibility of knowledge should influence context-sensitive reasoning. We examine this question by investigating the effects of time pressure on context-sensitive reasoning in the domain of biology. Biology provides an interesting domain in which to investigate this question for several reasons. First, there is evidence of context-sensitive reasoning in

P.Shafto, shafto@mit.edu 
biology among novice adults and children; reasoning about novel physiological properties like genes is guided by taxonomic knowledge exclusively whereas reasoning about novel diseases is also guided by causal/ecological knowledge (e.g. Coley et al., 2005; Shafto et al., 2005). Second, there is also evidence that although novice adults can demonstrate context-sensitive reasoning in some cases, reasoning based on taxonomic knowledge appears to be a robust default strategy, and taxonomic inferences are far more likely than causal/ecological inferences (Coley, Shafto, Stepanova, \& Baraff, 2005; López, Atran, Coley, Medin, \& Smith, 1997; Osherson, Smith, Wilke, Lopez, \& Shafir, 1990; Shafto \& Coley, 2003).

To examine the effects of knowledge accessibility on inductive reasoning, we employed a novel speeded/delayed inductive reasoning task. As in a standard category-based induction task, we taught a novel property about a premise category and queried participants about the likelihood that it would also be true of a conclusion category. We varied the property (gene versus disease) and the relations between premise and conclusion (taxonomic, ecological, or unrelated). Our innovation was to vary time pressure by manipulating how long participants had to make their inference. Participants were either speeded (urged to respond one second after an item appeared) or delayed (forced to wait $15 \mathrm{sec}$ before responding). Based on past research, we expected inferences to taxonomically related conclusions (hereafter, taxonomic inferences) to be rated more likely for novel genes than for novel diseases. Conversely, we expected inferences to ecologically related conclusions (hereafter, ecological inferences) to be rated more likely for novel diseases than for novel genes. We made two sets of related predictions about the effects of time pressure. First, time pressure should disproportionately affect inferences based on less accessible knowledge. If so, we expect ecological inferences to be rated less likely in the speeded conditions than in the delayed conditions, whereas taxonomic inferences and inferences to unrelated conclusions should be unaffected. Second, time pressure should disproportionately affect the context-sensitive use of less accessible knowledge to guide inferences. If so, we expect equivalent property effects on taxonomic inferences in both timing conditions, but more pronounced property effects on ecological inferences in the delayed condition.

\section{METHOD}

\section{Participants}

Sixty-four university undergraduates participated in exchange for course credit. Sixteen participants were randomly assigned to each of four conditions: speeded gene, delayed gene, speeded disease, delayed disease.

\section{Materials}

Materials consisted of 57 pairs of labeled photos of animals. Seventeen pairs were related taxonomically (same superordinate category, different habitat, e.g., tiger/camel), 15 were related ecologically (different superordinate category, same habitat, e.g., tiger/ parrot) and 25 were unrelated. Pictures were realistic photos of animals (with backgrounds removed) found on the Internet, and labeled with common folk-generic names. We used pictures combined with labels to maximize the likelihood that our biologically novice popu-
}

lation would recognize the species and therefore be able to access the relevant taxonomic and ecological knowledge.

\section{Design}

We manipulated three independent variables in the property induction task. Participants were randomly assigned to reason about novel genes or diseases, and to do so under speeded or delayed timing conditions. As described above, premise-conclusion pairs were related via taxonomy, ecology, or unrelated. Thus, the experimental design was a 2 (property: gene vs. disease) $\times 2$ (timing: speeded vs. delayed) $\times 3$ (item type: taxonomic vs. ecological vs. unrelated); property and timing were manipulated between subjects, and item type was manipulated within subjects.

\section{Procedure}

The experiment consisted of two parts: a property induction task followed by a belief-assessment task. Both parts of the experiment were conducted on a computer using PsyScope (Cohen, MacWhinney, Flatt, \& Provost, 1993). In the induction task, participants were shown all 57 items in random order, given information about one member of the pair, and asked about the second member. In the gene conditions, participants were asked, e.g., "Tigers have a gene. How likely is it that parrots have the same gene as tigers?" In the disease conditions, the word "disease" was substituted for "gene." Participants responded using a 1-7 scale, where 1 indicated "very unlikely" and 7 indicated "very likely."

Participants in the delayed condition were instructed to "Please take your time and think carefully about each item before typing your response. When the text turns white, you may enter your response." The question text initially appeared in red. After $15 \mathrm{sec}$, the text turned white. Participants were unable to enter their response until after the 15 -sec period had passed. Participants in the speeded condition were instructed, "When the text turns white, you may enter your response. Please try to respond as quickly as possibly after the text turns white, without sacrificing accuracy." The question text initially appeared in red, but turned white after only one second. The duration of the speeded condition was arrived at by considering an estimate of the minimal length of time it would take to read the question, fixations of about $250 \mathrm{msec} /$ word (Rayner, 1998), plus time to saccade, minus considerations for words that are repeated across trials. The delayed condition duration was then set to be long enough to allow reading the question and require participants to have a noticeable lag before responding. The difference in duration cannot guarantee that participants think about the questions more deeply in the delayed condition, of course, but it sets conditions that encourage or discourage detailed consideration of the problem.

In the belief-assessment task, participants were shown each item again in random order and asked "Do these animals live in the same habitat?" and "Do these animals belong to the same biological category?" They answered "yes," "no," or "don't know," but were instructed to use the final option sparingly. Participants responded "don't know" to a mean of 5.5 items for the ecological question $(9.7 \%)$ and 2.4 items for the taxonomic question (4.2\%).

\section{RESULTS}

Each participant was assigned three scores, reflecting average likelihood ratings for taxonomic, ecological, and unrelated items. These were submitted to a 2 (timing: delayed vs. speeded) $\times 3$ (item type: taxonomic vs. ecological vs. unrelated) $\times 2$ (property: disease vs. gene) mixed ANOVA. Overall, taxonomic inferences $(M=4.61)$ were rated more likely than ecological inferences $(M=3.25)$, which in turn were rated as more likely than unrelated inferences $\left[M=2.46, F(2,120)=197.24, M S_{\mathrm{e}}=0.385, p<\right.$ $\left..0001, p_{\text {rep }}>0.99, \eta_{p}^{2}=0.77\right] .{ }^{1}$ We also observed property effects for both taxonomic and ecological inferences, but 
not unrelated inferences $\left[F(2,120)=17.79, M S_{\mathrm{e}}=0.385\right.$, $\left.p<.0001, p_{\text {rep }}>0.99, \eta_{p}^{2}=0.22\right] .{ }^{2}$ As anticipated, taxonomic inferences were rated more likely for gene $(M=$ 4.95) than for disease $[M=4.27, t(62)=3.53, p=.0008$, $\left.p_{\text {rep }}=.99, d=0.44\right]$, whereas ecological inferences were rated more likely for disease $(M=3.56)$ than for gene [ $M=$ $\left.2.93 t(62)=2.96, p=.0043, p_{\text {rep }}=.97, d=0.38\right]$.

To test specific predictions about the effects of time pressure on context-sensitive reasoning we conducted 2 (property) $\times 2$ (timing) ANOVAs on mean likelihood ratings separately for taxonomic, ecological, and unrelated items. Results are presented in Figure 1. For taxonomically related items, as predicted, we observed no effect of time pressure; the finding reported above, that taxonomic inferences were stronger in the gene condition than the disease condition, held for both speeded and delayed conditions $[F(1,60)=$ $\left.12.45, M S_{\mathrm{e}}=0.591, p<.001, p_{\text {rep }}=.99, \eta_{p}^{2}=0.17\right] .{ }^{3}$

In contrast, for ecologically related items, as predicted, inferences were rated more likely in the delayed condition $(M=3.49)$ than in the speeded condition $(M=3.00$ $\left[F(1,60)=6.27, M S_{\mathrm{e}}=0.617, p=.015, p_{\text {rep }}=.4, \eta_{p}^{2}=\right.$ $0.09] .{ }^{4}$ Moreover, as predicted, property effects depended on timing. As seen in Figure 1, participants in the delayed conditions rated inferences to ecological matches as more likely for disease $(M=4.05)$ than gene $(M=2.93)$, whereas participants in the speeded conditions showed no difference $\left[M_{\text {disease }}=3.07, M_{\text {gene }}=2.92, F(1,60)=6.17\right.$, $\left.M S_{\mathrm{e}}=0.617, p=.016, p_{\text {rep }}=.94, \eta_{p}^{2}=0.09\right] .5$

For the unrelated items, as depicted in Figure 1, neither time pressure nor property influenced likelihood ratings, thereby demonstrating that delaying responses had a specific effect on the accessibility of ecological knowledge when relevant, rather than a general effect of increasing likelihood ratings across the board.

We also examined the degree to which beliefs about taxonomic and ecological relatedness of the stimulus pairs impacted inductive strength judgments. To do so, we excluded "don't know" responses and computed the proportion of positive responses to the questions about the taxonomic and ecological relatedness of each species pair. We also calculated the mean inductive strength score for each item, separately for each of the four groups of participants. We then conducted four multiple regression analyses by item, using beliefs about taxonomic and ecological relatedness to predict inductive strength in each of the four conditions. Standardized regression coefficients are presented in Figure 2.

All four regressions were highly significant. In both the speeded gene condition $\left[R^{2}=0.899, F(2,56)=241.35\right.$, $\left.M S_{\mathrm{e}}=0.20, p<.0001, p_{\text {rep }}>.99\right]$ and the speeded disease condition $\left[R^{2}=0.882, F(2,56)=202.26, M S_{\mathrm{e}}=0.15, p<\right.$ $\left..0001, p_{\text {rep }}>.99\right]$, inferences were based almost entirely on taxonomic knowledge (see Figure 2). In both speeded conditions, taxonomic relatedness showed a very strong predictive relation to inductive strength, and ecological relatedness made a much smaller, but nevertheless significant, contribution. In contrast, participants in the delayed conditions showed a clear pattern of differential recruitment of taxonomic and ecological knowledge based on property. In the delayed gene condition, taxonomic relatedness was highly predictive of inductive projections, whereas ecological re- latedness was unrelated to likelihood ratings $\left[R^{2}=0.908\right.$ $\left.F(2,56)=268.06, M S_{\mathrm{e}}=0.13, p<.0001, p_{\text {rep }}>.99\right]$. As seen in Figure 2, however, results were very different in the delayed disease condition; not only did both taxonomic and ecological relatedness make independent contributions to
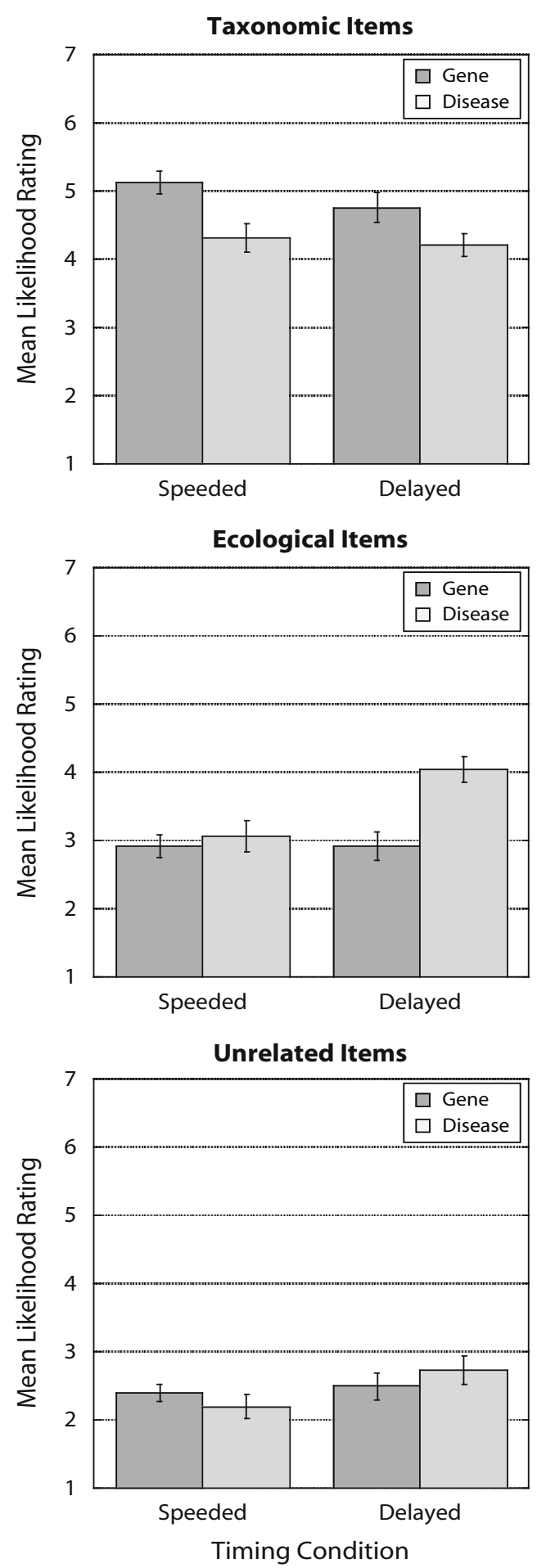

Figure 1. Effects of timing condition and property on mean likelihood ratings for inferences to taxonomic, ecological, and unrelated items. 


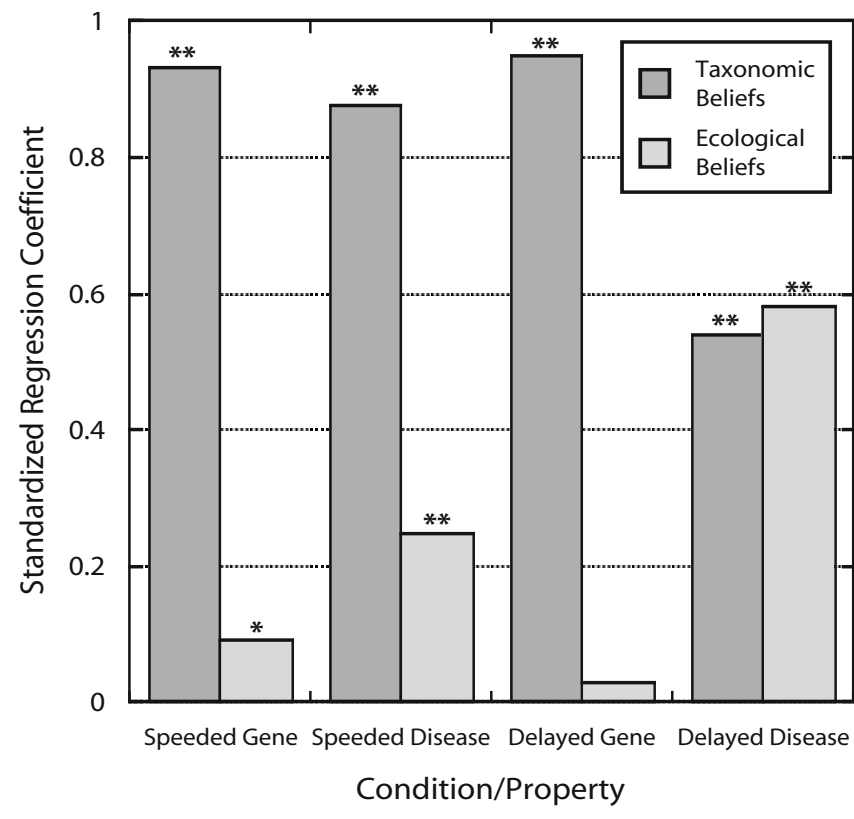

Figure 2. Standardized regression coefficients for predictive relations between beliefs about taxonomic and ecological relatedness and inductive strength by condition. $\quad * p<.05 . \quad{ }^{* *} p<.0001$.

explaining inductive strength ratings, but these contributions were of equivalent magnitude $\left[R^{2}=0.703, F(2,56)=\right.$ $\left.63.83, M S_{\mathrm{e}}=0.28, p<.0001, p_{\text {rep }}>.99\right]$.

\section{DISCUSSION}

Our results demonstrate the importance of knowledge accessibility for category-based property induction. For taxonomically based arguments, time pressure had no effect on rated argument strength, and we observed robust property effects suggesting an impact of the context of the inference. For ecologically based arguments, time pressure had a dramatic effect such that these arguments showed no property effects when responses were made under time pressure, but showed clear property effects when responses were delayed. Importantly, there were no effects of time pressure or property on unrelated items. These results were corroborated by regression analyses that showed when under time pressure, inferences about both genes and disease were overwhelmingly influenced by taxonomic knowledge, whereas under conditions in which participants were given time to consider the arguments, inferences about disease were equally informed by taxonomic and ecological knowledge.

These results are the first to our knowledge to address the time course of category-based induction. They show that relatively accessible taxonomic knowledge was used to guide inferences in a context-sensitive manner independent of time pressure, whereas context-sensitive use of less accessible ecological knowledge required additional time. Moreover, our results provide evidence that time pressure affects reasoning by restricting access to ecological knowledge; participants' beliefs about ecological relatedness predicted strength ratings much more strongly when reasoning about diseases in the delayed condition than in the speeded condition. Forcing participants to take more time to make decisions seems to have increased access to ecological knowledge allowing differential recruitment of knowledge as a function of the property being projected. Specifically, in the speeded condition, the relative contributions of taxonomic and ecological beliefs were the same for inferences about genes and disease. In contrast, in the delayed condition, inferences about genes were exclusively predicted by taxonomic beliefs whereas inferences about disease were equally informed by beliefs about taxonomic and ecological relatedness.

We have proposed that time pressure influenced reasoning by selectively affecting access to ecological knowledge. However, a possible alternative interpretation is that arguments that are relatively weak to begin with are simply weakened further when evaluated under time pressure. Specifically, taking the delayed condition as a baseline, is the decrease in ecological-disease ratings in the speeded condition attributable to the relatively weak baseline ratings, rather than to decreased access to ecological knowledge? 6 Our evidence suggests this is unlikely for two reasons. First, we observed no effects of time pressure on unrelated or taxonomic inferences, suggesting that only ecological inferences were rendered less likely in the speeded condition. Second, taxonomic and ecological inferences about disease received comparable likelihood ratings in the delayed condition ( $M=4.22$ and 4.05 , respectively). If time pressure further weakens arguments that are weak to begin with, then both taxonomic and ecological inferences about disease should be weaker in the speeded condition. However, only the ecological ratings decreased under time pressure, consistent with our argument that time pressure selectively affects access to knowledge.

Taken together, these results support the idea that knowledge accessibility is critical for inductive inference in general and for context-sensitive induction in particular and provide specific evidence that for these participants taxonomic knowledge was highly accessible whereas ecological knowledge, albeit present, was less accessible (Shafto, Coley, \& Vitkin, 2006). Furthermore, this work has provided a general method for investigating the relationship between knowledge and reasoning. By combining reasoning under time pressure with assessments of knowledge, we have shown how reasoning changes over time, and attributed those changes to differential access to knowledge. This combination provides a potentially useful tool for investigating how knowledge affects reasoning across experience, domains, and tasks.

These results are consistent with previous findings showing effects of knowledge accessibility on category use, and showing an advantage for taxonomic over other kinds of knowledge (e.g., Barsalou, 1982; Ross \& Murphy, 1999; Vitkin \& Coley, 2005). Specifically, inferences to taxonomically related species were rated more likely than inferences to ecologically related or unrelated species, and beliefs about the degree of taxonomic relatedness between premise and conclusion species were strongly predictive of reasoning in all conditions. These results underscore the salience and accessibility of taxonomic knowledge. We have also replicated previous studies showing that induc- 
tive inferences are sensitive to the property being projected (e.g., Heit \& Rubinstein, 1994; Ross \& Murphy, 1999); inferences to taxonomically related species were higher for gene than for disease, whereas the reverse was true for inferences to ecologically related species. However, our results also qualify previous demonstrations on inductive selectivity by showing how such selectivity is contingent on both possession of and access to relevant knowledge.

The present results raise questions about the effects of experience on accessibility of different kinds of knowledge. Several studies document increased use of ecological reasoning among biologically experienced populations relative to biologically naive undergraduates (e.g., Lopez et al., 1997; Proffitt et al., 2000; Shafto \& Coley, 2003). We have shown that ecological knowledge is less accessible than taxonomic knowledge among a biologically inexperienced population. It remains to be seen whether folk biological experience increases the accessibility of ecological knowledge; if so, we would expect effects of time pressure to disappear for experts. Alternatively, perhaps taxonomic knowledge remains relatively more accessible regardless of experience, and previous work with experts is equivalent to our delayed condition.

The present results also have implications for models of category-based reasoning and semantic knowledge. Specifically, models must take into account the impact of independent and dissociable kinds of knowledge on inductive inference, and moreover, differences in relative accessibility of that knowledge. Approaches based on structured theories may provide a framework in which to explain these effects (Shafto, Kemp, Baraff, et al., 2005; Shafto, Kemp, Bonawitz, et al., 2005; Shafto et al., 2006; Tenenbaum, Kemp, \& Shafto, in press). These models posit separable knowledge structures that explain reasoning in different contexts, but because they are computational-level models do not address processing issues such as the time course of accessing knowledge.

In conclusion, we have begun to explore the time course of category-based induction. We have shown that time pressure influences the context-sensitive use of ecological knowledge in induction, whereas the context-sensitive use of taxonomic knowledge was impervious to time pressure. We have argued that these results demonstrate the impact of differential accessibility of ecological and taxonomic knowledge about animals on inductive inferences. This work contributes both a set of theoretical and empirical constraints on viable accounts on human inductive reasoning, and a novel methodology to further explore those constraints.

\section{AUTHOR NOTE}

This material is based on work supported by National Science Foundation Grant BCS-0236338 to J.D.C. We thank Anna Vitkin for helpful comments on an earlier draft. Correspondence regarding this article should be addressed to P. Shafto, Department of Brain and Cognitive Sciences, Massachusetts Institute of Technology, 77 Massachusetts Avenue, Cambridge, MA 02139 (e-mail: shafto@mit.edu).

\section{REFERENCES}

Barsalou, L. W. (1982). Context-independent and context-dependent information in concepts. Memory \& Cognition, 10, 82-93.
Cohen, J. D., MacWhinney, B., Flatt, M., \& Provost, J. (1993). PsyScope: A new graphic interactive environment for designing psychology experiments. Behavior Research Methods, Instruments, \& Computers, 25, 257-271.

Coley, J. D., Shafto, P., Stepanova, O., \& Baraff, E. (2005). Knowledge and category-based induction. In W. Ahn, R. L. Goldstone, B. C. Love, A. B. Markman, \& P. Wolff (Eds.), Categorization inside and outside the laboratory: Essays in honor of Douglas L. Medin (pp. 69-85). Washington, DC: American Psychological Association.

Coley, J. D., Vitkin, A. Z., Seaton, C. E., \& Yopchick, J. E. (2005). Effects of experience on relational inferences in children: The case of folk biology. In B. G. Bara, L. Barsalou, \& M. Bucciarelli (Eds.), Proceedings of the 27th Annual Conference of the Cognitive Science Society (pp. 471-475). Mahwah NJ: Erlbaum.

Heit, E., \& Rubinstein, J. (1994). Similarity and property effects in inductive reasoning. Journal of Experimental Psychology: Learning, Memory, \& Cognition, 20, 411-422.

López, A., Atran, S., Coley, J. D., Medin, D., \& Smith, E. E. (1997). The tree of life: Universal and cultural features of folkbiological taxonomies and inductions. Cognitive Psychology, 32, 251-295.

Osherson, D. N., Smith, E. E., Wilkie, O., Lopez, A., \& Shafir, E. (1990). Category-based induction. Psychological Review, 97, 185-200.

Proffitt, J. B., Coley, J. D., \& Medin, D. L. (2000). Expertise and category-based induction. Journal of Experimental Psychology: Learning, Memory, \& Cognition, 26, 811-828.

RAYNER, K. (1998). Eye movements in reading and information processing: 20 years of research. Psychological Bulletin, 124, 372-422.

Ross, B. H., \& MurPHy, G. L. (1999) Food for thought: Cross-classification and category organization in a complex real world domain. Cognitive Psychology, 38, 495-553.

Shafto, P., \& Coley, J. D. (2003). Development of categorization and reasoning in the natural world: Undergraduates to experts, naive similarity to ecological knowledge. Journal of Experimental Psychology: Learning, Memory, \& Cognition, 29, 641-649.

Shafto, P., Coley, J. D., \& Vitkin, A. (in press). Availability in category-based induction. In A. Feeney \& E. Heit (Eds.), Inductive reasoning: Cognitive, mathematical and neuroscientific approaches. Cambridge: Cambridge University Press.

Shafto, P., Kemp, C., Baraff, E., Coley, J. D., \& Tenenbaum, J. B. (2005). Context-sensitive reasoning. In Proceedings of the 27th Annual Conference of the Cognitive Science Society (pp. 2003-2008). Mahwah, NJ: Erlbaum.

Shafto, P., Kemp, C., Bonawitz, E., Coley, J. D., \& Tenenbaum, J. B. (2005). Inductive reasoning about causally transmitted properties. Manuscript submitted for publication.

Shafto, P., Kemp, C., MansinghKa, V. K., Gordon, M., \& TenenBAUM, J. B. (2006). Learning cross-cutting systems of categories. In Proceedings of the 28th Annual Meeting of the Cognitive Science Society (pp. 2146-2151).

Tenenbaum, J. B., Kemp, C., \& Shafto, P. (in press). Theory-based Bayesian models of inductive reasoning. In A. Feeney \& E. Heit (Eds.), Induction. Cambridge: Cambridge University Press.

Vitkin, A., \& Coley, J. D. (2005, November). Accessibility of taxonomic and script knowledge in the domain of food. Paper presented at the 46th Annual Meeting of the Psychonomic Society, Toronto.

\section{NOTES}

1. This effect was also significant by items $\left[F(2,54)=74.59, M S_{\mathrm{e}}=\right.$ $\left.1.259, p<.0001, p_{\text {rep }}>.99, \eta_{p}^{2}=0.73\right]$.

2. By items $\left[F(2,54)=40.91, M S_{\mathrm{e}}=0.168, p<.0001, p_{\text {rep }}>.99\right.$, $\eta_{p}^{2}=0.60$.

3. By items $\left[F(1,16)=45.29, M S_{\mathrm{e}}=0.173, \mathrm{p}<.0001, p_{\text {rep }}>.99\right.$, $\eta_{p}^{2}=0.73$.

4. By items $\left[F(1,14)=24.28, M S_{\mathrm{e}}=0.149, p=.0002, p_{\text {rep }}=.99\right.$, $\eta_{p}^{2}=0.63$.

5. By items $\left[F(1,14)=11.27, M S_{\mathrm{e}}=0.316, p=.0047, p_{\text {rep }}=.97\right.$, $\eta_{p}^{2}=0.44$.

6 . We thank a reviewer for pointing this out.

(Manuscript received September 4, 2006; revision accepted for publication November 7, 2006.) 